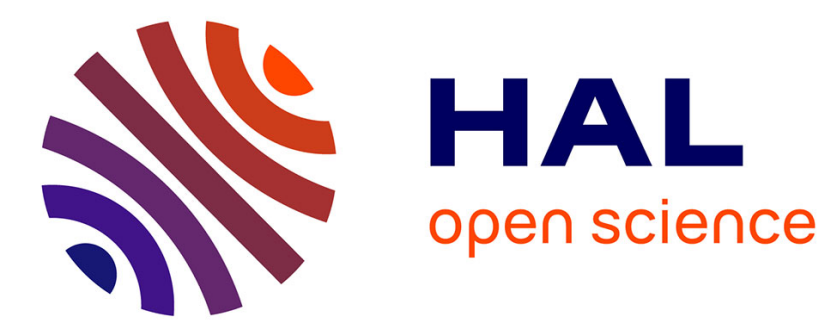

\title{
De la lutte armée à la lutte électorale, itinéraires divergents d'une trajectoire insolite, une comparaison à partir des cas centraméricains et colombien
}

David Garibay

\section{- To cite this version:}

David Garibay. De la lutte armée à la lutte électorale, itinéraires divergents d'une trajectoire insolite, une comparaison à partir des cas centraméricains et colombien. Revue internationale de politique comparée, 2005, Vol. 12 ( $\mathrm{N}^{\circ} 3$ ), pp.283-297. halshs-00136364

\section{HAL Id: halshs-00136364 \\ https://shs.hal.science/halshs-00136364}

Submitted on 9 Feb 2008

HAL is a multi-disciplinary open access archive for the deposit and dissemination of scientific research documents, whether they are published or not. The documents may come from teaching and research institutions in France or abroad, or from public or private research centers.
L'archive ouverte pluridisciplinaire HAL, est destinée au dépôt et à la diffusion de documents scientifiques de niveau recherche, publiés ou non, émanant des établissements d'enseignement et de recherche français ou étrangers, des laboratoires publics ou privés. 


\author{
David Garibay \\ Maître de conférences en science politique \\ Université Lumière Lyon 2 - CNRS UMR 5206 Triangle
}

De la lutte armée à la lutte électorale, itinéraires divergents d'une trajectoire insolite. Une comparaison à partir des cas centraméricains et colombien

Article publié in

Revue internationale de politique comparée

Vol 12, 2005/3, p.283-297

De manière assez paradoxale, l'analyse de l'évolution d'organisations de guérillas en partis politiques, dans le cadre de processus de paix négociés incluant des éléments de démocratisation, apparaît comme un thème marginal dans la recherche sur les liens entre les partis politiques et la violence armée [1]. Pourtant dans une réflexion générale sur la situation de la gauche en Amérique latine, ces expériences témoignent de l'itinéraire particulier, et relativement insolite, de partis de gauche, remarquable à la fois par leur modalité d'insertion dans les institutions et par le fait qu'ils revendiquent une continuité avec un projet de changement radical. Dans la majeure partie de la région, la gauche partisane se restructure à l'occasion des transitions démocratiques après une longue période d'interdiction, de répression et d'exil. Son intégration aux institutions politiques se réalise par la lutte électorale, syndicale ou sociale. Or "l'expérience de la gauche dans les pays d'Amérique centrale et en Colombie a été radicalement différente de celle du reste de l'Amérique latine" [2]. En effet, sa trajectoire est fondée davantage sur le recours à la violence armée, et aboutit à une négociation dans une situation de conflit qui permet la légalisation de la guérilla en parti politique en même temps que sa démobilisation.

Dans de nombreux pays, des dirigeants et militants de mouvements de gauche ont participé à des expériences de lutte armée. Mais leur abandon de la guérilla et leur incorporation dans des partis a été généralement le fait de décisions individuelles, liées d'abord à la répression par les forces armées, et ensuite aux amnisties concédées aux opposants pendant les transitions démocratiques. Hormis le cas de certaines fractions du Partido de los Trabalhadores (PT) au Brésil, du Frente Amplio en Uruguay, et dans une moindre mesure, du Partido de la Revolución Democrática (PRD) au Mexique, ces incorporations n'ont pas donné lieu à l'existence institutionnelle de tendances, qui, au sein des partis de gauche, revendiquent une continuité avec le moment de la lutte armée.

De ce fait, après l'expérience de la transformation des Fuerzas Armadas para la Liberación Nacional dans le Movimiento al Socialismo (MAS) au Venezuela en 1971 [3], les exemples récents de la transformation de guérillas en partis politiques en Colombie, au Salvador et au Guatemala apparaissent bien comme des expériences de cette trajectoire insolite de la lutte armée à la lutte électorale [4]. En 1990 en Colombie, le Movimiento 19 de Abril (M-19) constitue la Alianza Democrática M-19 (ADM-19). Il est suivi par l'Ejército Popular de Liberación (EPL), le Partido Revolucionario de los Trabajadores (PRT), et dans une dynamique plus autonome, par la guérilla indigène du Movimiento Armado Quintín Lame [5]. Les Accords de paix signés en 1992 au Salvador prévoient la fin du conflit armé, et entre autres choses, la démilitarisation du Frente Farabundo Martí para la Liberación Nacional (FMLN) et sa légalisation en parti politique. Un processus assez semblable conduit à la signature en 1996 des Accords de paix au Guatemala, permettant la légalisation de la Unión Revolucionaria Nacional Guatemalteca (URNG). 
Le bilan électoral de ces partis semble pour le moins diversifié. Au Venezuela, le MAS a maintenu dans les décennies 1970 et 1980 un discours de gauche innovant dans le contexte partisan de ce pays, mais il n'obtient jamais plus de 6\% des voix jusqu'à 1988. Dans le contexte de crise du système bipartisan, il dépasse régulièrement $10 \%$ aux élections entre 1988 et 1998, appuie en 1993 la coalition hétéroclite autour de la candidature à la Présidence de l'ancien dirigeant démocrate-chrétien Rafael Caldera et participe, à la suite de la victoire de ce dernier, au gouvernement. Le soutien critique à Hugo Chávez depuis 1998 conduit néanmoins le parti à une perte de spécificité. En Colombie, la ADM-19 connaît un succès aussi fulgurant qu'éphémère. Elle obtient $12,5 \%$ à la présidentielle de mai 1990, et 26,7\% lors de l'élection de décembre 1990 pour l'Assemblée constituante. Mais en octobre 1991, elle ne rallie plus que $9,4 \%$ des voix aux élections législatives, puis $3,8 \%$ à la présidentielle de mai 1994, scrutin qui marque quasi-ment la fin de l'organisation. Au Guatemala, l'URNG n'est jamais parvenue à une audience électorale autre que symbolique, victime de ses propres divisions et de son incapacité à fédérer une gauche guatémaltèque largement atomisée. Dès les élections de 1999, elle se divise, un secteur appuyant une alliance de gauche dont le candidat obtient $12,3 \%$ à la présidentielle, un autre se présentant seul et ne ralliant que $1,6 \%$. Le résultat obtenu en 2003 ( 2,6\%) confirme sa marginalisation. De fait, le cas du Salvador apparaît comme le seul exemple de réussite électorale et partisane consolidée dans le temps d'une formation issue d'une guérilla démobilisée à la suite d'un accord négocié [6] : crédité de $25 \%$ à la présidentielle et de $21 \%$ aux législatives, en 1994, lors de sa première participation à des élections, le FMLN n'a depuis cessé de progresser. Aux élections présidentielles, il est toujours distancé par le parti au pouvoir ( $57,7 \%$ pour le candidat de Alianza Republicana Nacionalista - ARENA - contre 35,7\% à celui du FMLN lors de la présidentielle de mars 2004). Mais il rallie de manière régulière près d'un tiers des voix aux élections législatives, où il a même dépassé son principal rival ( $34,0 \%$ pour le FMLN contre $31,9 \%$ pour ARENA aux législatives de 2003 ). Il a par ailleurs conquis et conservé de très nombreuses municipalités, et tout particulièrement la capitale et ses environs.

Ces fortunes électorales diverses témoignent du fait qu'il n'est en aucune manière possible de traduire automatiquement une capacité militaire, et bien souvent un soutien significatif auprès de la population civile lors de la période de conflit interne, en un appui électoral une fois la paix signée. Inversement, la confrontation dans les urnes ne signifie pas pour autant la disparition programmée de partis qui dans leur ensemble se revendiquent en continuité avec l'histoire et surtout avec l'orientation idéologique de l'organisation révolutionnaire armée dont ils sont issus [7]. Bien davantage que leur force militaire, ou même leur capacité à imposer, dans la négociation, des revendications qui leur soient prioritaires, la réussite des anciennes organisations de guérilla à devenir des partis de gauche, à la fois structurés en interne, revendiquant une position de changement radical, et consolidés dans leur influence, réside plutôt dans leur capacité à se déconnecter du contexte spécifique de leur transformation et à s'insérer dans des clivages partisans nationaux. Ce processus suppose un changement, où les références internes liées à la période de la lutte armée s'estompent progressivement, sans être pour autant définitivement éliminées, au profit de celles de la lutte électorale. Il conduit également, en cas de succès, à faire de la formation en question la principale organisation de la gauche partisane dans chaque pays, en marginalisant d'autres options, dans un contexte de forte polarisation.

De guérilla à parti, une métamorphose inédite

La transformation de guérilla en partis politiques à l'issue d'un processus négocié a constitué un événement inédit sur le plan historique. Hormis les exemples de victoires révolutionnaires à Cuba en 1959 et du Nicaragua en 1979, les autres formations constituées en Amérique latine 
ont été détruites par les forces de l'ordre, assez rapidement dans la plupart des cas de foyers insurrectionnels des années 1960, mais aussi à l'issue de conflits prolongés comme dans le cas péruvien des années 1980. Si l'on exclut le cas très spécifique de la guérilla néo-zapatiste au Mexique, seules survivent aujourd'hui comme guérillas combattantes les deux organisations colombiennes des Fuerzas Armadas Revolucionarias de Colombia (FARC) et du Ejército de Liberación Nacional (ELN).

En faisant le choix des armes, les guérillas se positionnent elles-mêmes devant l'alternative qui devint le slogan des révolutions triomphantes, "la victoire ou la mort". Par la nature du conflit armé entre guérillas et gouvernements, l'éventualité de la négociation ne participe pas à l'univers des possibles. Elle apparaît en effet, pour les premières, comme la conservation du régime contre lequel elles luttent, c'est-à-dire la négation même de leur projet révolutionnaire, et pour les seconds comme la reconnaissance de la violence armée comme un moyen, sinon légitime, du moins valide pour obtenir la satisfaction de revendications radicales sur la scène institutionnelle. Cette position intransigeante, revendiquée de part et d'autre, distingue le recours aux armes par les mouvements révolutionnaires des déstabilisations par la violence des régimes libéraux par leurs opposants, tentatives qui ont émaillé l'histoire républicaine des États latino-américains depuis leur indépendance. Le développement de guérillas révolutionnaires, comme recours spécifique à la violence armée, a fait l'objet d'une analyse comparée en profondeur, dans laquelle ont été interrogées les conditions de leur surgissement, de leur capacité à se maintenir dans le temps et enfin, les raisons de leur succès dans deux cas ou de leur échec ailleurs à renverser les régimes qu'elles contestaient [8]. Dans les cas spécifiques des pays d'Amérique centrale et de la Colombie, le développement dans la durée de la lutte armée a longtemps obéré la consolidation de partis politiques de gauche, à la fois par le ralliement des cadres de ces partis, lorsqu'ils existaient, à la voie des armes, et par l'extension de la répression par les forces armées contre l'ensemble de la gauche.

Or la difficulté de penser la possibilité de la transformation de guérillas en partis politiques dans le contexte d'une transition démocratique négociée incluant la résolution d'un conflit armé interne a été renforcée par les modalités des changements institutionnels qu'ont connu les autres pays d'Amérique du sud dans les années 1980. La quasi-absence de groupes armés, la faiblesse de la gauche révolutionnaire, tout autant que le contrôle exercé par les militaires sur les négociations ont conduit à interpréter ces processus comme devant être en même temps pacifiques et modérés. Le modèle de transition démocratique qui en est issu repose en effet sur une double condition : l'exclusion des acteurs armés, parce que le recours à la violence altère d'emblée la conduite des négociations, et la valorisation de la modération des acteurs présents, puisque toute revendication radicale, en particulier de la gauche, peut créer des résistances au changement au sein du régime autoritaire. Un tel paradigme explicatif s'avérait rapidement limité pour des cas où des acteurs radicaux avaient fait explicitement le choix des armes [9], et où la possibilité même d'une "transition insurgée" surgissait à la fois de l'importance de la mobilisation sociale et des effets d'un conflit armé prolongé, et non d'une volonté d'ouverture contrôlée du régime autoritaire [10].

Cette difficulté semblait pouvoir être levée par les leçons tirées du cas vénézuélien et des premières tentatives de négociation menées entre guérillas et gouvernement dans les années 1980, qu'il s'agisse des dialogues initiés en Amérique centrale lors du processus d'Esquipulas ou des accords de trêve signés par le gouvernement Betancur en Colombie en 1984. Plutôt que sur les dynamiques d'une transition démocratique, l'accent est mis alors sur le fait que certaines réformes politiques peuvent inciter à la recherche d'une solution négociée, en facilitant l'accès aux institutions représentatives. L'analyse est dès lors portée sur les choix stratégiques des guérillas face aux possibilités d'expression politique offertes par les gouvernements dans le cadre de dialogues nationaux [11]. L'échec des dialogues en Colombie, la difficile poursuite de négociations en Amérique centrale, mais également la 
comparaison avec certains cas d'Afrique australe [12], conduisent à prolonger cette réflexion sur la possibilité de la transformation des guérillas en partis politiques. Dans une perspective néo-institutionnaliste, certains auteurs insistent sur la relation entre coût de suppression de l'opposition armée et coût de tolérance d'une opposition partisane issue d'une guérilla démobilisée - ce dernier étant lié aux types de réformes qui permettraient de limiter l'influence électorale éventuelle de ces organisations [13].

En élargissant cette perspective à l'analyse de la manière dont les processus de transition démocratique affectent les comportements des acteurs qui y participent, il est possible de montrer combien les évolutions institutionnelles dans le sens d'une ouverture démocratique, sans restriction aux seules élections, ont modifié l'itinéraire suivi par les groupes révolutionnaires de l'action armée vers trois scénarios possibles : un arrangement concerté, une reddition négociée ou une disparition du mouvement. Dans ce contexte, plus que la force militaire, le facteur explicatif de la réussite de cette intégration réside dans l'importance des soutiens, politiques et sociaux, que peuvent recevoir les guérillas en cours de démobilisation [14].

Mais avec l'accomplissement des processus de paix en Amérique centrale, l'analyse comparée s'est davantage centrée sur les modalités de la négociation, et en particulier sur l'élucidation de cas, finalement assez peu fréquents, de situations dans lesquelles une intervention multilatérale très importante a été la garantie non seulement de la signature de la paix mais également de l'absence de reprise des hostilités armées [15]. En se centrant sur le processus de paix en tant que tel, cette perspective a mis l'accent, d'une part sur les dynamiques internes de la négociation, de l'autre sur l'accomplissement des modalités contenues dans les accords, et en particulier sur les manières de penser une éventuelle rediscussion lors de la mise en œuvre sans remettre en cause l'esprit initial du texte. Cette approche conduit néanmoins à minimiser l'importance de la question de la transformation des organisations armées en partis politiques à l'issue de leur démobilisation comme un facteur de stabilisation du processus dans son ensemble. L'importance accordée à cet aspect semble être à l' aune de la place extrêmement limitée que les différents accords de paix allouent au devenir des partis issu des guérillas. Dans tous les cas considérés ici, les textes négociés portent essentiellement sur les conditions de la démilitarisation, tant de la guérilla que de l'armée, sur les programmes de réinsertion, sur certaines réformes institutionnelles et sur la réconciliation nationale. Il y est mentionné, de manière très ponctuelle, qu'à l'issue de leur démobilisation, les anciennes organisations armées pourront être légalisées en partis politiques. Mais aucune disposition spécifique ne vient organiser leur première participation aux élections, qui doit dès lors se réaliser selon les mêmes conditions que tout autre parti [16].

L'importance de la participation des partis issus des anciennes guérillas a été reconsidérée dans les travaux qui insistent sur le fait que les cas comparés relèvent de situations où la résolution du conflit interne et la démocratisation ne sont pas deux dimensions parallèles, mais bien des dynamiques entremêlées dont l'effective réalisation est réciproque et conditionne en grande partie l'évolution des régimes. La réintroduction d'une dimension temporelle plus longue que celle de la stricte négociation [17], l'interrogation sur le comportement des élites et sur la dimension des changements structurels introduits par ces accords [18], le questionnement sur le caractère hybride des régimes nés de ces négociations, du fait de la distance entre l'avancée formelle des droits politiques et leur application réelle [19], permettent de montrer combien dans ces cas "l'obtention d'une paix longue et durable n'est possible que si la principale cause des guerres civiles y est résolue, c'est-à-dire si les changements institutionnels entendent répondre à l'imperméabilité du système politique aux demandes sociales. Ainsi les pactes de paix établissent des compromis qui permettent l'intégration des demandes des guérillas dans le système représentatif (ce qui suppose dès lors leur reconversion en partis politiques)" [20]. Dans les "transitions insurgées", où 
démocratisation et résolution négociée des conflits internes s'accompagnent, à l'image des cas centraméricains et colombien, le devenir des organisations politiques issues des guérillas démobilisées n'est pas qu'un résultat contingent, mais apparaît bien comme un des éléments d'évaluation de la consolidation dans le temps de la réussite à la fois du processus de paix et de la démocratisation.

\section{Continuités et disparitions des formations partisanes issues des guérillas}

L'émergence de partis politiques de gauche issus de guérillas démobilisées constitue bien une nouveauté, non seulement dans l'histoire des pays concernés, mais plus généralement dans l'ensemble de la région comme modalité d'apparition de partis de gauche. Ce qui paraît remarquable dans cette intégration est que ces formations vont revendiquer une continuité avec l'organisation armée dont elles sont issues : cela les conduit dès lors à assumer, dans un premier temps, à la fois l'héritage de la période de la lutte armée et celle de la négociation, et va se traduire, à moyen terme, par le maintien d'une revendication idéologique et programmatique de changement radical. Or les systèmes politiques nationaux, tant en Colombie qu'en Amérique centrale ne se caractérisent pas jusqu'alors par le fait de permettre l'expression de tels positionnements. Lorsqu'elle existe, la réussite électorale de ces partis en est d'autant plus remarquable. Éphémère en Colombie, elle repose sur l'assimilation entre radicalité armée et volonté rapide de changement institutionnel, c'est-à-dire sur la fusion du projet de l'organisation dans l'exceptionnelle temporalité politique de la Constituante. Plus progressive, mais beaucoup plus consolidée dans le temps au Salvador, la réussite du FMLN tient à la capacité de construire une organisation partisane à partir d'un positionnement très à gauche, avec une forte homogénéité interne, tant des électeurs que des cadres, et un contenu programmatique renouvelé par rapport à celui du contexte initial de la transition.

La continuité entre l'organisation qui a mené la lutte armée et le parti qui en est issu constitue un élément interne qui distingue particulièrement ces formations d'autres partis. Les relations internes au sein d'une guérilla, façonnées par le compartimentage vertical et la clandestinité, les fidélités à la hiérarchie militaire et à l'organisation, les normes de valorisation interne des militants, fondées sur le courage physique, l'héroïsme, et le dévouement à la cause, le charisme du commandement - mais également le fait de subsumer toute revendication à l'objectif de la victoire militaire - sont autant de normes internes qui apparaissent rapidement en contradiction avec l'idée de constituer un appareil partisan au fonctionnement interne ouvert, transparent, pluraliste et démocratique.

Si la revendication de la continuité avec l'organisation armée est extrêmement présente, elle se réalise néanmoins selon des modalités qui façonnent d'emblée l'émergence de nouveaux modes de fonctionnement internes. Ce lien est particulièrement affiché dans ce qui relève de l'apparence externe de l'organisation : la décision de conserver le nom et les principaux symboles au sein du nouveau parti témoigne de cette volonté de continuité. Dans la manière de présenter leur histoire, la période de la lutte armée est systématiquement revendiquée comme une étape de la formation de ces partis.

En dépit du fait qu'elle intègre par la suite d'autres organisations, la ADM-19 colombienne conserve le nom du M-19, ses couleurs et donne une place particulière dans sa symbologie au premier et au dernier de ses commandants militaires. Dans les cas du FMLN au Salvador et de l'URNG au Guatemala, la conservation du nom et des symboles est manifeste dès le congrès constitutif du parti : elle s'accompagne néanmoins d'une mutation particulièrement représentative du changement en cours. Dans ces deux pays, c'est le nom de l'organisation d'ensemble qui est revendiqué, alors même que les fonctions de celle-ci ont été limitées pendant la période du conflit armé à la direction plus ou moins coordonnée et à la représentation diplomatique de différents groupes de guérillas très jaloux de leur autonomie 
interne et de leur identité militante (cinq au Salvador, trois au Guatemala). Il n'y avait pas de ce fait de dirigeant unique qui puisse prétendre à lui seul représenter l'ensemble de l'organisation. Ce sont les différents groupes qui ont fait la guerre sur le terrain, mais c'est au nom de leur représentation commune que la paix a été négociée et signée et que la lutte électorale va être menée. De fait, les deux partis se structurent initialement en reproduisant à l'identique les composantes internes, dans l'autonomie est respectée, et en adoptant des principes de représentation paritaire par organisation. Or ils vont connaître un processus rapide d'institutionnalisation vers une structure unitaire, avec l'effacement des anciennes composantes, et l'émergence de logiques majoritaires dans l'élection des directions, tout en conservant, comme légat de cette période, l'absence d'un chef unique.

Ce transfert d'identifications politiques des différentes composantes vers une seule structure n'est pas sans conséquences, tant les rivalités internes pouvaient être aiguës. Au Guatemala, il va renforcer au sein de l'URNG les tendances centrifuges. Au Salvador, par contre, malgré de très sérieuses tensions internes, il va permettre que les références aux organisations de guérilla s'estompent progressivement au profit de l'identité de l'organisation commune, favoriser l'éclosion de nouvelles lignes de clivage et l'intégration de nouveaux adhérents qui n'ont pas participé à la lutte armée. Dans les débats internes sur l'orientation programmatique et stratégique du parti, deux camps principaux s'opposent, les tenants d'un affichage plus à gauche et d'une démarche centrée sur le parti et les partisans d'une ligne plus réformiste, davantage ouverts à des alliances. Ce clivage traverse les anciennes identités organisationnelles. Preuve de l'émergence de ces nouveaux clivages, le rappel d'une légitimité militante gagnée dans la lutte armée comme ressource pour s'imposer à des postes de direction au sein du parti est de moins en moins invoqué

Pour autant, ces évolutions internes paraissent moins déterminantes dans le devenir de l'organisation que la dynamique qui se réalise entre le contexte particulier dans lequel elle est devenue un parti politique et la revendication du maintien d'une ligne de changement radical. Le cas colombien présente une caractéristique qui le différencie des situations centraméricaines : devant la quasi-absence d'accord de paix entre le gouvernement et le M19, ce dernier va investir la Constituante comme lieu de l'élaboration de ce qui n'a pas été négocié lors de sa démobilisation [21]. De ce fait, l'ADM-19 apparaît sans doute comme l'exemple le plus abouti d'une réussite dans le moment d'intégration du mouvement dans la scène politique, mais d'un échec à poursuivre au-delà de ce contexte initial [22]. Or dans ce succès initial, il convient de comprendre la manière dont l'organisation assume l'héritage du M-19, et prétend en incarner la volonté de changement radical. Ce groupe avait voulu se distinguer des clivages habituels de la gauche révolutionnaire et revendiquait un positionnement original, justifiant le choix des armes par une conception volontariste de la nécessité d'un changement, au nom des visions bolivariennes des héros de l'indépendance, d'un nationalisme progressiste et social, et d'un approfondissement des pratiques démocratiques des institutions du pays. La radicalité n'est pas tant présente dans le contenu du projet politique que dans les modalités pour y parvenir. Or la direction de l'organisation réussit totalement à adapter cette vision, davantage fondée sur des principes d'action que sur des postulats idéologiques, au contexte très particulier de la Colombie des années 1990-1991. La volonté explicite de recherche du dialogue entre tous et de la représentation de la nation dans sa diversité se cristallise dans un premier temps dans la constitution d'une liste très ouverte pour l'élection à la Constituante, puis surtout dans l'esprit réformateur, pluriel, consensuel et innovateur qui se dégage de cette Assemblée. L'osmose est telle que l'organisation se confondra avec ce moment particulier de la vie politique colombienne, et ne survivra pas à la fin de l'exceptionnelle période de changement, en ne parvenant pas à renouveler son discours radical au-delà de l'incantation rhétorique sur la nécessité des réformes et sur la nécessité du dialogue. 
À l'image du cas vénézuélien, les deux cas centraméricains relèvent plus classiquement de l'apparition de formations revendiquant un positionnement affiché à l'extrême gauche de l'échiquier politique, où la radicalité renvoie à un contenu idéologique et programmatique. Le MAS se définissait à son origine comme un mouvement communiste non aligné sur Moscou. Lors de sa constitution en parti, en 1995, le FMLN se présente comme "un parti démocratique et révolutionnaire de lutte permanente, qui cherche à regrouper et défendre les intérêts des grandes majorités populaires", éléments auxquels, il ajoute, en 1997, l'adjectif "socialiste" et une revendication d'une perspective de genre.

Pourtant les contextes politiques nationaux apparaissent particulièrement défavorables à l'émergence de forces politiques se revendiquant ouvertement à gauche. Tant au Salvador qu'au Guatemala, avec des configurations internes différentes, c'est largement sur la répression de la gauche politique qu'ont reposé à la fois les formules autoritaires antérieures (qu'il s'agisse de la Matanza de 1931 au Salvador, ou de la révolte militaire qui mit fin au gouvernement de Jacobo Arbenz en 1954 au Guatemala) et celles qui ont caractérisé la période du conflit interne de la décennie 1980. La fin de la période de négociation avait permis en pratique la participation politique du centre-gauche, mais ce n'est qu'avec les Accords de paix de 1992 au Salvador et de 1996 au Guatemala et la transformation des guérillas en partis que les élections deviennent totalement pluralistes.

Or la nouvelle conjoncture ouvre en réalité un autre défi à la gauche centraméricaine, celui de trouver face à elle, dans la lutte électorale, des formations de droite au pouvoir, solidement structurées, qui ont évolué d'un positionnement d'extrême droite militariste à un alignement sur des orientations néolibérales, et bénéficient d'un important soutien du gouvernement des États-Unis [23]. L'affichage à gauche de ces nouvelles formations en est d'autant plus remarquable. Les partis issus de la guérilla s'insèrent dans un clivage idéologique fortement marqué, construit sur une polarisation de l'affrontement électoral, à la fois intense et pacifique. Or l'effet de polarisation entre différentes options idéologiques va conduire à la déconnexion par rapport au moment de la négociation des accords de paix et de la rhétorique de la réconciliation nationale. Dans ce cadre, le contenu en matière de politiques sectorielles des textes des accords participe à définir la marge d'action plus ou moins grande de ces nouveaux partis [24]. Au Guatemala, l'Accord de paix de 1996 ratifie d'importantes réformes en matière sociale, en particulier en ce qui concerne les droits des populations indigènes (même si ceux-ci demeurent largement formels). L'URNG se trouve ainsi privée de revendications qu'elle considère comme spécifiques. Cette situation ne fait qu'alimenter la crise interne de l'organisation et la conduit rapidement à la marginalisation électorale.

Au contraire au Salvador, l'Accord de paix de 1992 est beaucoup plus restreint aux seules dimensions institutionnelles. Il permet au FMLN de se présenter comme un parti ayant un projet idéologique bien déterminé, qu'il est le seul à défendre. Dans ce contexte, non seulement le parti perdure, mais il occupe une position hégémonique à la gauche du panorama partisan. De fortes tensions internes ont certes conduit à plusieurs reprises à des scissions de ses dirigeants les plus modérés [25], mais cela a contribué également à renforcer à la fois l'homogénéité interne et une position idéologique plus radicale, sans affecter ni sa position dominante dans la gauche, ni remis en question son emprise électorale. Malgré plusieurs tentatives, aucune formation de centre-gauche n'est parvenue à un résultat électoral significatif [26]. Le FMLN a obtenu autour de $34 \%$ des voix aux trois élections législatives ( 1997,2000,2003). Si lors des élections présidentielles, il continue à être fortement distancé par le parti au pouvoir, son résultat est néanmoins conforme à son influence ( $29,0 \%$ en $1999,35,7 \%$ en 2004) [27]. Au niveau local, il gouverne plus de 70 municipalités, dont une proportion très élevée des villes les plus peuplées - parmi elles, 5 capitales départementales et 11 des 14 villes de la zone métropolitaine de la capitale sans discontinuité depuis 1997. La consolidation électorale du parti à un niveau important repose sur un électorat homogène, 
fortement urbanisé, concentré dans les classes moyennes et populaires, avec un niveau élevé de conscience politique et une auto-identification fortement marquée à gauche. Ce profil fortement idéologisé correspond à celui de ses élus [28].

Si les revendications programmatiques du parti répondent à un positionnement sur le clivage droite/gauche, cette polarisation renvoie néanmoins largement à un effet de miroir face à un gouvernement qui affiche ouvertement ses orientations économiques néo-libérales et son alignement politique sur Washington. En réclamant le retour à la monnaie nationale, le FMLN critique ainsi la politique de "dollarisation", symbole du sacrifice de la souveraineté nationale. En accompagnant la très importante mobilisation sociale dans le secteur de la santé en 2003 contre le projet gouvernemental de privatisation, le parti affiche son opposition aux politiques de libéralisation et dérégulation, tout en pratiquant une relation nouvelle avec des mouvements sociaux, autrefois inféodés à l'objectif révolutionnaire et désormais largement autonomes. Or dans ce discours de critique, les références révolutionnaires et les liens autrefois affichés avec les "partis frères" de l'ancien Bloc de l'est sont définitivement remplacés par de nouveaux modèles internationaux et surtout latino-américains. Des représentants du PT brésilien étaient présents lors de l'investiture du nouveau maire de la capitale en 2003, des dirigeants du PRD mexicain ont participé à la campagne présidentielle de 2004. Dans la critique virulente à l'appui donné par le gouvernement à l'intervention armée en Irak ou à la signature d'un accord de libre-échange avec les États-Unis, les rhétoriques anti-impérialistes se nourrissent désormais de références nouvelles à la légalité internationale, dans le premier cas, et d'exemples d'autres pays de la région, capables de négocier même dans une situation asymétrique, dans le second. Symbole de ces mutations, le programme du parti lors de la campagne présidentielle de 2004 s'ouvrait par un extrait du Programme du Millénaire des Nations-Unies. À ce renouvellement des références répond également l'émergence progressive d'une nouvelle génération de cadres dirigeants, dont la légitimation interne provient désormais d'une action municipale, caractérisée davantage par son pragmatisme et sa volonté de transparence que par sa radicalité programmatique [29].

Le cas salvadorien apparaît comme l'exemple le plus abouti non seulement de transformation en parti mais également de réussite électorale d'une formation issue de la démobilisation de guérillas dans le cadre de processus négociés incluant une démocratisation des institutions. Malgré les limites des autres cas, il signale plus globalement un mode particulier d'apparition de partis de gauche dans les panoramas partisans d'Amérique latine depuis les années 1980. Or, la spécificité ne réside pas seulement dans les modalités de négociation. Alors que les transitions concertées d'Amérique du sud ont conduit généralement à l'émergence de clivages partisans nouveaux et mouvants, les "transitions insurgées" ont eu pour résultat d'institutionnaliser le clivage idéologique qui était au cœur du conflit armé, et dès lors de permettre l'expression d'une polarisation importante - l'existence d'un débat politique vif et animé n'ayant pas pour autant conduit au danger d'une reprise des hostilités. Dans le reste de la région, les partis de gauche ont généralement recherché, pour consolider leurs résultats, voire pour parvenir au pouvoir, à modérer leur positionnement et à établir des alliances, durables ou conjoncturelles, avec des formations de centre, voire de centre-droit. Cela leur a effectivement permis d'accéder au pouvoir, d'abord au niveau local, puis au niveau central, mais ne s'est pas systématiquement traduit par un maintien ou une augmentation de leurs résultats électoraux à un niveau élevé. Dans ce contexte, le FMLN apparaît véritablement comme un cas à part : il est en même temps aujourd'hui l'un des partis de gauche qui obtient sur un temps relativement long les résultats électoraux les plus élevés, avec des performances semblables à tous les niveaux d'élection, tout en étant l'un de ceux dont les positionnements à gauche sont les plus affirmés.

Annexe : Liste des sigles 
ADM-19 : Alianza Democrática M-19 (Colombie) : parti issu de la démobilisation du M-19, créé en 1990

ARENA : Alianza Republicana Nacionalista (Salvador) : parti politique (droite), créé en 1982, au pouvoir depuis 1989

CDU : Centro Democrático Unido (Salvador): parti politique (centregauche), créé en 1998

ELN : Ejército de Liberación Nacional (Colombie): guérilla, créée en 1964, initialement d'orientation guévariste, toujours en activité

FARC : Fuerzas Armadas Revolucionarias de Colombia : guérilla d'orientation communiste, créée en 1966, toujours en activité

FMLN : Frente Farabundo Martí de Liberación Nacional (Salvador) : organe de coordination des cinq groupes de guérilla, créé en 1980, démobilisé en 1992, devenu parti politique

FSLN : Frente Sandinista de Liberación Nacional (Nicaragua) : organe de coordination des trois groupes de guérilla, créé en 1960, au pouvoir de 1979 à 1989, dans l'opposition depuis.

M-19 : Movimiento 19 de Abril (Colombie): guérilla créée en 1970, démobilisée en 1990

MAS : Movimiento al Socialismo (Venezuela): parti politique (gauche) issu de la démobilisation de la guérilla

PRD : Partido de la Revolución Democrática (Mexique): parti politique (gauche)

PT : Partido de los Trabalhadores (Brésil) : parti politique (gauche)

URNG : Unión Revolucionaria Nacional Guatemalteca (Guatemala): organe de coordination des trois groupes de guérilla, démobilisé en 1996, devenu parti politique.

\section{NOTES}

[1] Longtemps marqués par un angle d'approche cherchant à élucider le choix de la violence armée par un groupe politique légal, les questionnements dans ce domaine abordent récemment l'usage simultané par un même groupe du recours à la violence et de la présence dans l'arène institutionnelle. WEINBERG L. and PEDAHZUR A., Political Parties and Terrorist Groups, Londres, Routledge, 2003

[2] ANGELL A., "Incorporating the Left Into Democratic Politics", part II, p. 20, in DOMINGUEZ J., LOWENTHAL A., Constructing Democratic Governance, Baltimore, The Johns Hopkins University Press, 1996.

[3] ELLNER S., Venezuela's Movimiento al Socialismo, Durham, Duke University Press, 1988.

[4] L'expérience du Nicaragua a été volontairement exclue de cette réflexion comparée sur les partis de gauche provenant de la lutte armée. Si le Frente Sandinista de Liberación Nacional (FSLN) a effectivement connu une longue période de lutte armée, il a également gouverné pendant toute la période sandiniste ( 1979-1989) comme parti-État. De même, l'expérience en Colombie de la Unión Patriótica, constituée par les FARC au moment des accords de trêve de 1984 n'est pas non plus abordée, car elle ne se réalise pas à la suite de la démobilisation de la guérilla, mais comme la volonté d'utiliser la trêve pour constituer un parti politique.

[5] Pour l'ensemble des groupes, PEÑARANDA R., GUERRERO J., De las armas a la política, Bogota, Tercer Mundo, IEPRI, 1999. Pour le mouvement indigène, LAURENT V., Communautés indiennes et espaces politiques en Colombie, Thèse de sociologie, Université de Paris 3,2001

[6] MASSÉ F., "De la violencia a lo político, una reconversión lograda ?", Análisis Político, 36, janvier 1999 .

[7] Dans leur étude sur l'explication de l'avènement ou non de révolutions dans le Tiers Monde, Jeff Goodwin et Theda Skocpol, lorsqu'ils évoquent les organisations armées, affirment que "l'urne électorale peut ne pas être le cercueil de la conscience de classe (...) 
mais elle a prouvé qu'elle était celui des mouvements révolutionnaires" in GOODWIN J., SKOCPOL T., "Explaining Revolutions in the Contemporary Third World", Politics and Society, 17,4,1989, p. 495 .

[8] WICKHAM-CROWLEY T., Exploring Revolution, Essays on Latin American Insurgency and revolutionary theory, London, Sharpe, 1991 et WICKHAM-CROWLEY T., Guerrillas and revolution in Latin America, Princeton, Princeton University Press, 1992.

[9] DOMINGUEZ J., "Democratic transitions in Central America and Panama" in DOMINGUEZ J., LINDENBERG M., Democratic transitions in Central America, Gainesville, University Press of Florida, 1997.

[10] Sur la critique de l'argument de la modération, BERMEO N., "Myths of Moderation, Confrontation and Conflict during Democratic Transitions", Comparative Politics, 29,3,1997 ; sur le rôle de la mobilisation sociale dans les transitions démocratiques, COLLIER R., Paths toward Democracy, Cambridge, Cambridge University Press, 1999; sur l'idée de "transitions insurgées", avec une analyse comparée appliquée à l'Afrique du Sud et au Salvador, WOOD E. J., Forging Democracy from Below, Cambridge, Cambridge University Press, 2000.

[11] En 1986, les présidents centraméricains se réunissent à Esquipulas (Guatemala) et s'engagent à mettre en œuvre des dialogues avec les mouvements armés. Pour sa part, Belisario Betancur, élu Président de Colombie en 1982, mène une politique d'ouverture envers les guérillas, qui conduit à la signature d'accords de trêve et à des dialogues nationaux. DEAS M., "The colombian peace process 1982-85 and its implication for Central America" in DI PALMA G., WHITEHEAD L., The Central AmericanImpasse, Londres, Croom Helm, 1986 et CHERNICK M., "Negociated Settlement to Armed Conflict : Lessons from the Colombian Peace Process", Journal of Interamerican Studies and World Affairs, 30,4, Hiver 1988-89.

[12] La résolution négociée de la rébellion armée des mouvements africains en Rhodésie du sud et la transformation de la guérilla en parti unique lors de la création du Zimbabwe en 1979 constitue une référence pour les acteurs en conflit en Colombie et en Amérique centrale dans les années 1980. Mais c'est surtout la recherche des solutions négociées aux conflits internes en Angola ( 1991,1994) et au Mozambique ( 1992), puis dans la corne de l'Afrique et dans une certaine mesure la fin de l'apartheid en Afrique du sud qui vont nourrir la réflexion comparée. Sur le Zimbabwe, GRIFFITH A., Conflict and Resolution : Peace Building Through the Ballot Box in Zimbabwe. Oxford, New Cherwell Press, 1998. Sur les cas africains, MESSIANT C., MARCHAL R., Les chemins de la guerre et de la paix. Paris, Karthala, 1997. Sur la question spécifique de la transformation d'un ancien mouvement armé en parti politique, pour le cas de la RENAMO au Mozambique, CAHEN, M., Les Bandits, Paris, C. Gulbekian, 2002.

[13] SHUGART M. S., "Guerrillas and Elections : an Institutionnalist Perspective on the Costs of Conflict and Competition", International Studies Quarterly, 36,1992.

[14] RYAN J., "The Impact of Democratization on Revolutionnary Movements", Comparative Politics, 27,1, octobre 1994. Pour une application au cas colombien, BOUDON L., "Guerrillas and the State : the Role of the State in the Colombian Peace Process", Journal of Latin American Studies, 28,2, mai 1996.

[15] BEJARANO J. A., Una Agenda para la paz, Bogotá, Tercer Mundo, 1995, ARNSON C., Comparative Peace Processes in Latin America, Washington, Wilson Center Press, 1999 et MONTGOMERY T. S., Peacemaking and Democratization in the Western Hemisphere, Coral Glabes, North South Center Press, 2000.

[16] En Amérique centrale, les États-Unis ont incité, durant la décennie 1980, à l'organisation d'élections pour permettre l'émergence de pouvoirs civils. Les Accords de paix au Salvador en 1992 et au Guatemala en 1996 ne prévoient généralement que l'amélioration du fonctionnement des tribunaux électoraux, mais ne modifient ni les modes de scrutin, ni les 
calendriers. C'est donc dans les conditions et les calendriers établis dans les années 1980 que les organisations issues des guérillas participent aux élections, en 1994 au Salvador et en 1999 au Guatemala. En Colombie, dans la négociation avec le M-19, l'accord avait prévu un système de "favorabilidad política" qui garantissait des sièges aux anciennes guérillas lors de leur première participation aux élections. Le rejet de cet accord par le Congrès a rendu cette disposition caduque (voir note 21 ).

[17] CALVERT P., "The Democratic Transition in Central America", Conflict Studies, 307, mars 1998.

[18] BERNTZEN E., "Democratic Consolidation in Central America : a Qualitative Comparative Approach", Third World Quarterly, 14,3,1993.

[19] KARL T. L., "The Hybrid Regimes of Central America", Journal of Democracy, 6,3, juillet 1995

[20] DUCATENZEILER G., CHINCHILLA F., "Pactos de paz : proceso de pacificación y transición hacia la democracia en El Salvador y Guatemala", Revista de Ciencias Sociales, 12,2001, p. 168.

[21] Les négociations entre le gouvernement et le M-19 commencent en janvier 1989. L'accord final qui prévoyait d'importants changements institutionnels est néanmoins rejeté par le Congrès en décembre 1989. Le M-19 décide malgré cela de maintenir sa démobilisation, effective en mars 1990, et limitée en réalité à la remise de ses armes et à une amnistie. Un programme de réinsertion des anciens guérilleros est négocié postérieurement.

[22] ZULUAGA J., "De guerrillas a movimientos políticos, Análisis de la experiencia colombiana, el caso del M-19", in PEÑARANDA R., GUERRERO J., De las armas a la política, op. cit. et BOUDON L., "Colombia's M-19 Democratic Alliance : a Case-Study in New-Party Self-Destruction", Latin American Perspectives, 28, 1, Janvier 2001.

[23] MARTI S., "La izquierda centroamericana, renacimiento o debacle ?", pp. 65-108, in CARDENAL A. S., MARTI S., América Central, las democracias inciertas, Madrid, Tecnos, 1998.

[24] Sur cette idée, DUCATENZEILER G., CHINCHILLA F., "Pactos de paz : proceso de pacificación y transición hacia la democracia en El Salvador y Guatemala", op. cit., 2001.

[25] Les scissions les plus visibles sont celle de 1994, où, à l'issue des premières élections auxquelles le FMLN participe, un tiers de son groupe parlementaire fait scission pour constituer une formation plus modérée, et celles de 2002-03, pendant lesquelles des dirigeants modérés quittent le parti, et tout particulièrement Héctor Silva, maire de San Salvador de 1997 à 2003.

[26] C'est ainsi que le Centro Democrático Unido, la principale formation de centre-gauche, n'a jamais réussi à dépasser $6,5 \%$ aux élections législatives. Aux présidentielles, il a rassemblé 7,5\% des voix en 1999, mais seulement 3,9\% en 2004 .

[27] Pour une analyse des dernières élections (législatives et municipales de 2003, présidentielle de 2004), ZAMORA R., "El Salvador en la encrucijada, alternabilidad o continuidad ?", Nueva Sociedad, 186, 2003, ARTIGA A., "El Salvador. Maremoto electoral en 2004", Nueva Sociedad, 192, juillet 2004 et GARIBAY D., "Salvador, les défis du renouvellement. Le FMLN et les élections de 2003 et 2004", Problèmes d'Amérique latine, 54 , octobre 2004.

[28] Les résultats de l'enquête auprès des élites parlementaires menée par l'Université de Salamanque montrent un fort positionnement à gauche des députés du FMLN : en 1997-2000, ils se plaçaient eux-mêmes en moyenne en 1,9 sur une échelle gauche-droite de 1 à 10 , ce qui constitue l'un des positionnements les plus à gauche de la région. Sur les enquêtes électorales et le positionnement des députés du FMLN, ARTIGA A., "El Salvador", in ALCANTARA M., FREIDENBERG F., Partidos políticos de América Latina, Centroamérica, México y República Dominicana, Salamanca, Editorial Universidad de Salamanca, 2001. 
[29] Sur le gouvernement local du FMLN, DIAZ N., "El buen gobierno, el reto de la izquierda en El Salvador", p. 111-132, in STOLOWICZ B., Gobiernos de izquierda en América Latina, el desafío del cambio, Mexico, UAM, Plaza y Valdes, 2001. 